\title{
Design and Development of Microcontroller Remote Controlled Airport Luggage Cart
}

\author{
Mary Charlemaine A. Abas ${ }^{1, *}$, Patrick D. Cerna ${ }^{2}$ \\ ${ }^{1}$ Department of Electronics Technology, Federal Technological Institute University, Addis Ababa, Ethiopia \\ ${ }^{2}$ Department of Information Technology, Federal Technological Institute University, Addis Ababa, Ethiopia \\ *Corresponding author: engr.marycharlemaineabas@yahoo.com
}

\begin{abstract}
Nowadays, people love to travel anywhere. Airports mainly never run out of bustling crowds of traveler. They are carrying heavy luggage which is one of the biggest travel hassle. It is indispensable that airports offer luggage carts to ease such burden. Theses luggage carts are largely found in airports, large bus stations, hotels, or train stations. However, extra effort is required to navigate especially those individual with disability or the elders. Thus, the researchers proposed a remote controlled luggage cart. The main objective of this study is to create a luggage cart that being controlled by the owner by using a remote control. Specifically, it aims the following: (1) to construct a luggage cart that can carry less than or equal to 5.6 kilograms weight of luggage; (2) to wirelessly control the luggage cart using two Arduino Microcontrollers, two Zigbee modules, a direction/tilt sensor; and (3) to navigate the wheels of the luggage cart using a relay, dc motor and servo motor. This luggage cart is limited to stop, forward, left and right directions, as well as limited turning. It has its own specific frequency dedicated to follow its owner.
\end{abstract}

Keywords: Arduino, luggage, remote control, robot, airport

Cite This Article: Mary Charlemaine A. Abas, and Patrick D. Cerna, "Design and Development of Microcontroller Remote Controlled Airport Luggage Cart." International Transaction of Electrical and Computer Engineers System, vol. 4, no. 2 (2017): 49-54. doi: 10.12691/iteces-4-2-1.

\section{Background of the Study}

Airport is one of the crowded places in the world. Plane is usually the primary transportation for business trips, family vacations, or studying abroad. Thus, it is necessary that airports provide luggage carts for travelers to ease the burden of traversing from one terminal to another or around the airport areas.

Baggage carts, luggage carts, luggage trolleys or trolleys are small vehicles manually navigated by travelers. There are two major sizes: One for big luggage and one for small luggage. Carts have usually two parts for carrying luggage: a small section (basket) for carry-on luggage at the same level as the handle, and a lowered large section for suitcases and a small or large bag [1].

Nowadays, technology has greatly influenced airports as well. Remote controlled luggage cart are being introduced like the NUA Robotics that is mainly controlled by connecting to the owner's smart phone. It uses proximity sensors to prevent collision with other things.

The researchers proposed a design where the luggage cart does not need any human effort. This is a remote controlled luggage cart managed by the owner using Arduino and Zigbee technologies. There will be a remote control given to the owner to navigate such luggage cart. The luggage cart will automatically follow the direction of the owner's action using the remote control.
The main objective of this design is to create a luggage cart that being controlled by the owner by using a remote control. Specifically, it aims the following: (1) to construct a luggage cart that can carry less than or equal to 5.6 kilograms weight of luggage; (2) to wirelessly control the luggage cart using two Arduino Microcontrollers, two Zigbee modules, a direction/tilt sensor; and (3) to navigate the wheels of the luggage cart using a relay, dc motor and servo motor.

\section{Related Literature and Studies}

\subsection{Cart-A-Long}

Cart-A-Long is an improved shopping cart that automatically follows the user. It can be used not just in grocery or shopping but also in carrying luggage in airports. It uses an infrared (IR) sensor that locates the infrared emitter carried by the user as a bracelet to determine his angle and distance. The two infrared sensors were mounted on the stepper motors to control it. When the IR sensors established connection with the IR emitter, the IR sensor will give a feedback to the Arduino to control the stepper motors that would eventually control the two wheels at the rear of the cart. After receiving the feedback from the IR sensors to the first Arduino, it will give feedback to the second Arduino to control the DC motor. This DC motor will control the third front swivel wheel [2]. 


\subsection{NUA Robotics}

NUA Robotics is a mini robotic suitcase that follows the owner. Through connecting to the user's smartphone, the suitcase will know where to go and it gives the user information like location and weight of the suitcase. It uses proximity sensor to detect obstacles to ensure its safety. Its operating hours is from 60-90 minutes. This technology is still being finalized [3].

\subsection{Wireless Controlled Luggage Carrier}

This device named "The Stalker" is a hands-free load carrying propulsion system that maintains a safe following distance behind the user. The basic design functionality of the stalker entails wireless connectivity to the user. The wireless tethering is accomplished through the use of sensors on a bracelet/strap worn by the user, and sensors on the stalker unit [4].

\subsection{Self-Propelled Luggage}

Self-Propelled luggage is a device with a propelling system that can be controlled remotely, and a sensor system to help to navigate the container. The self-propelled/motorized luggage shall track and follow the user's motion and direction as guided by a remote control, smart phone and foot sensor. A distance can range from 1 to several feet in radius [4]. The control can be set to a follow-me mode in which the luggage propelling system will simply follow the remote using sensor signals transmitted and received between the remote and the transceiver in the luggage propelling system. In another embodiment, the control can be set to a control mode in which the remote can be used to direct the luggage propelling system to move the luggage from one point to the next [5].

\subsection{Related Empirical Studies}

Kannan and Selvakumar [6] design a Voice Controlled Robot (VCR) is a mobile robot whose motions can be controlled by the user by giving specific voice commands. The speech is received by a microphone and processed by the voice module. When a command for the robot is recognized, then voice module sends a command message to the robot's microcontroller.

Aneida and Hormos [7] developed a robot robotic module based on Arduino Uno technology. Communication between the robotic module and the operator (an end-user device) is done via a WiFi network for operation and control purposes. This communication occurs over a secured custom-made Encryption/Decryption Algorithm to prevent others from controlling the module. The distance between the module and the operator depends on the adapter itself. A camera "eye of robot" captures and transmits images/videos to the operator who can then recognize the surrounding environment and remotely control the module.

Cuasito [8] developed aEducational and Entertainment Mobile Robots Utilizing Arduino Microcontroller. In this design, the basic criteria sets the prototype to be light weight, "Arduino" microcontroller-based, programmable in C-Language, powered by internal battery, and a choice of DC or servo motor prime movers. The control designs are guided by the discrete truth table where input and output devices interact via programming through appropriate electronic interfacing.

Similarly, Balasubramanian et al. [9] studied an efficient mechanisms using Arduino to control robots. In this study, joystick controlled robot, mobile controlled robot and laptop controlled robot are manually controlled robot while the obstacle avoider robot and line follower robot are automatically controlled using proximity sensors. All the robots discussed above have same Arduino board, L293D and motor driving unit but only the types of controlling the robot varies.

Summarizing the literature, it is evident that there has been no attempt or scant study in the application of microcontroller in airport luggage cart robotic automation

\section{Materials and Methods}

\subsection{Materials Used in the Research Study}

The proposed remote controlled airport luggage cart utilizes Arduino Mega microcontroller with its accompanying shields, sensors, and modules as shown in Table 1.

Table 1. List of Materials

\begin{tabular}{|l|c|}
\hline \multicolumn{1}{|c|}{ Items } & Quantity \\
\hline Arduino Mega 2560 & 1 \\
\hline Directional/Tilt Sensor & 1 \\
\hline DC Motor & 2 \\
\hline TowerPro MG-995 Digital Servo & 1 \\
\hline Relay & 2 \\
\hline Wheel & 4 \\
\hline Female to Male connecting wires & 10 \\
\hline Zigbee Module & 2 \\
\hline
\end{tabular}

\section{Arduino Mega 2560}

The Mega 2560 is a microcontroller board based on the ATmega 2560. It has 54 digital input/output pins (of which 15 can be used as PWM outputs), 16 analog inputs, 4 UARTs (hardware serial ports), a $16 \mathrm{MHz}$ crystal oscillator, a USB connection, a power jack, an ICSP header, and a reset button. It contains everything to support the microcontroller; simply connect it to a computer with a USB cable or power it with an AC-to-DC adapter or battery to get started. The Mega 2560 board is compatible with most shields designed for the Uno and the former boards Duemilanove or Diecimila [10].

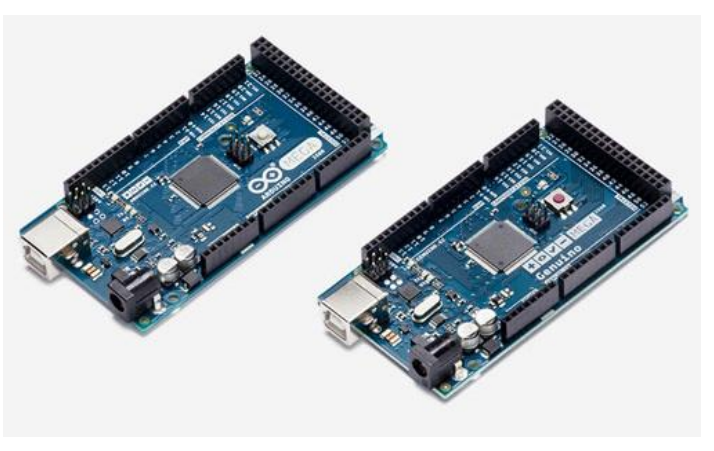

Figure 1. Arduino Mega 2560 Controller 


\section{Zigbee shield}

The Zigbee shield allows an Arduino board to communicate wirelessly. It is based on the Xbee module from MaxStream. The module can communicate up to 100 feet indoors or 300 feet outdoors (with line-of-sight). It can be used as a serial/USB replacement or placing it into a command mode and configuring it for a variety of broadcast and mesh networking options. The shields break out each of the Xbee's pins to a through-hole solder pad. It also provides female pin headers for use of digital pins 2 to 7 and the analog inputs, which are covered by the shield [11].

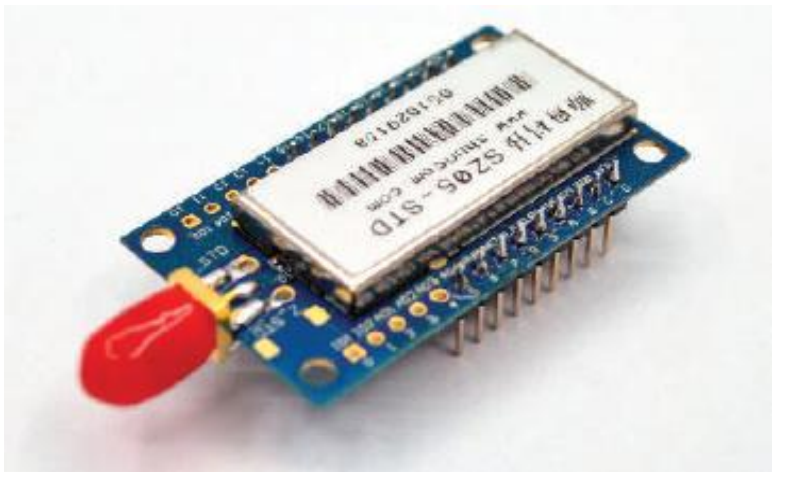

Figure 2. Zibbee Module

\section{Tilt Sensor}

A direction/tilt sensor is a type of sensor that has four-directional tilt detection. It is programmed using a microcontroller and has only a positive and a negative $\mathrm{x}$ and y-axes. This is commonly used in smart phones to change the screen display once the device is rotated [12].

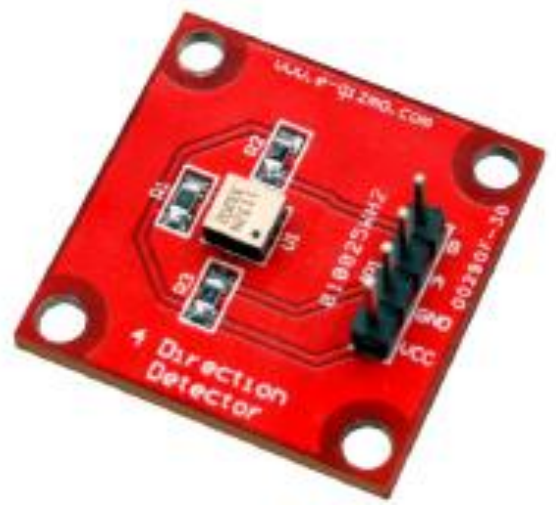

Figure 3. Direction Tilt Sensor

\section{Servo Motor}

A servo motor is a rotary or linear actuator that precisely controls the angular or linear position, velocity and acceleration. It has a motor connected to a sensor for position feedback. It receives control signals that would dictate the position of the servo shaft and a power applied to its DC motor to turn the shaft to that position [13].

Relay

A relay is an electronic device that acts as a switch. It opens or closes circuits electromechanically or electronically. It controls one electrical circuit by opening and closing in another circuit. When a relay contact is normally open (NO), there is an open contact when the relay is not energized. Same goes with a relay contact that is normally closed (NC). But when an electrical current is applied to the contacts, it will change their state [14].

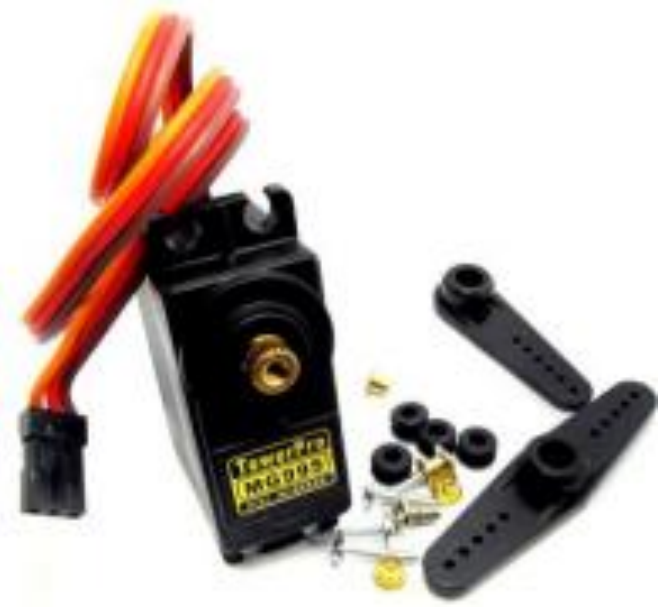

Figure 4. Servo Motor TowerPro MG-995

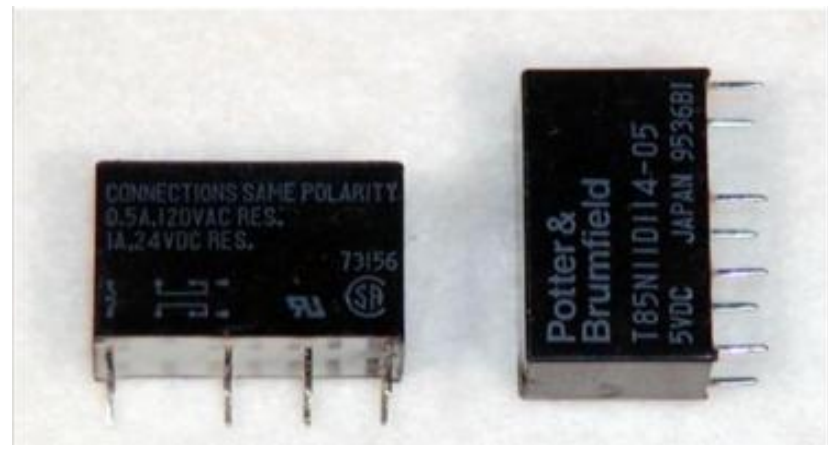

Figure 5. Double Pole Double Throw (DPDT) Mount Relay

\subsection{Methods and Design}

Figure 6 shows the system flowchart of the design. The directional/tilt sensor is with the owner and once the owner moves, it will detect its movement. The data gathered from the tilt sensor will be processed using the Arduino Mega. The processed data will be transmitted using a zigbee module and will be received by the luggage cart using anotherzigbee module. The received data will again be processed using another Arduino Mega. When the data of the $\mathrm{x}$ - axis is greater than the threshold specified by the user, the DC motor will move forward, otherwise, it will stop. When the data of the y-axis is greater than the threshold specified by the user, the servo motor will move to the right, otherwise, to the left.

Figure 7 shows that circuit diagram of the remote control user device. This controls the direction of the luggage cart. Likewise, Figure 8 reflects the circuit diagram of the luggage cart with the Arduino Mega micro controller, Zigbee module, DC motors and relays.

\subsection{Standard Procedures}

Objective 1: To contruct a luggage cart that can carry less than or equal to 5.6 kilograms weight of luggage.

1. Cut plywood with a dimension of $28 \times 18.5 \times 12$ inches.

2. Mount two wheels at the back of the plywood that are connected to a DC motor.

3. Mount another two wheels in a shaft in front of the plywood where the shaft is connected to a servo motor. 


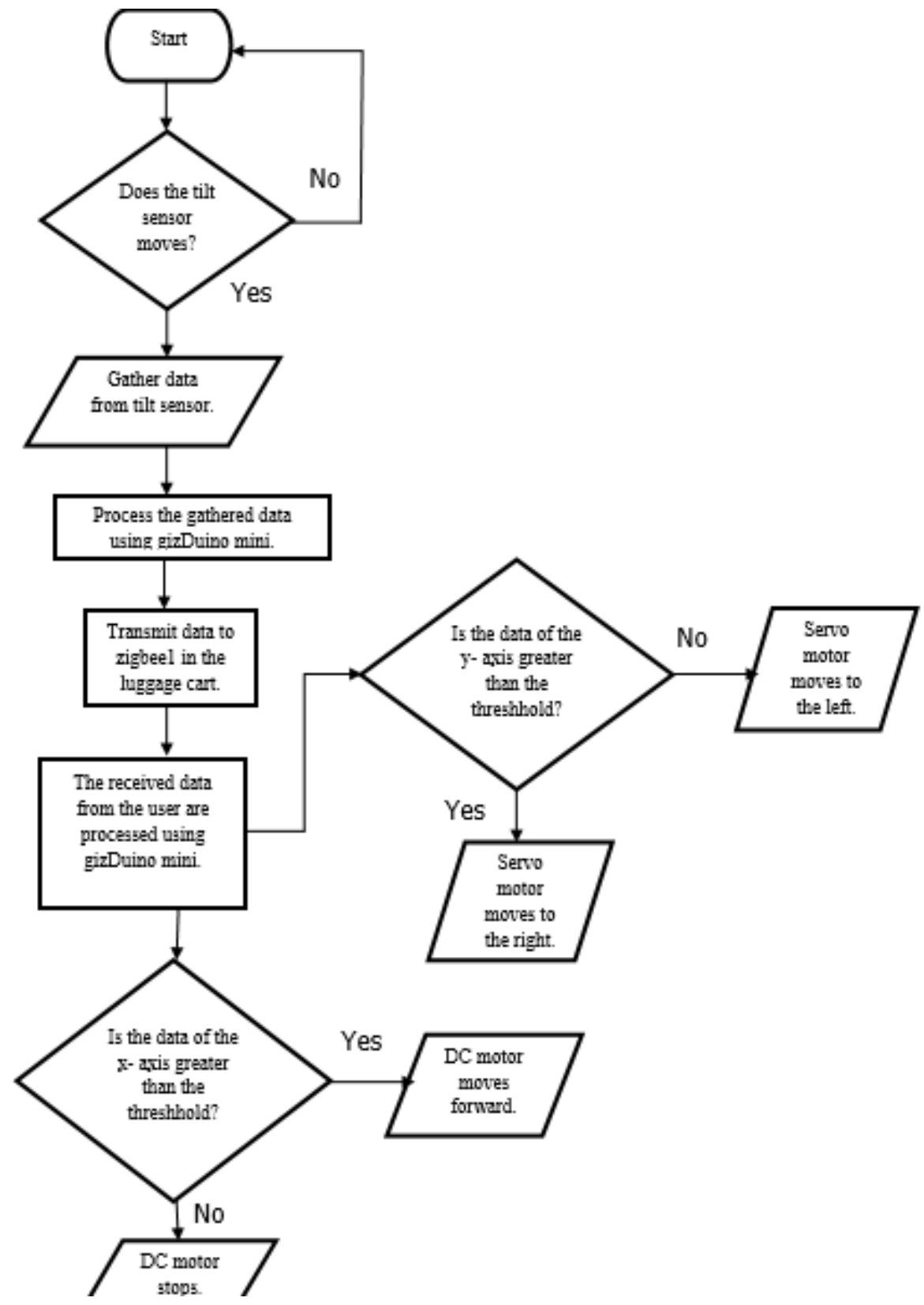

Figure 6. System Flowchart of the Design

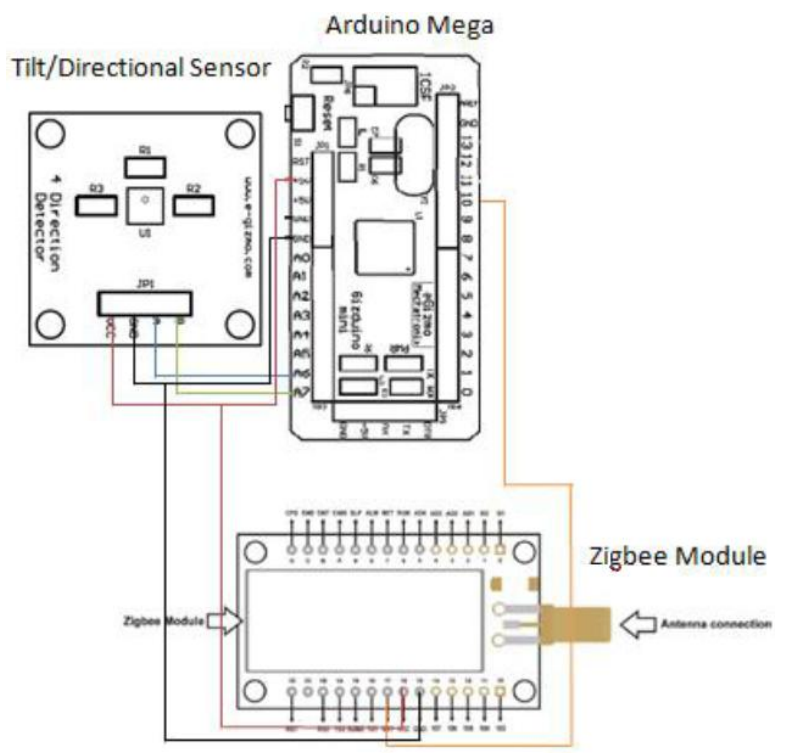

Figure 7. Circuit Diagram of the User Device

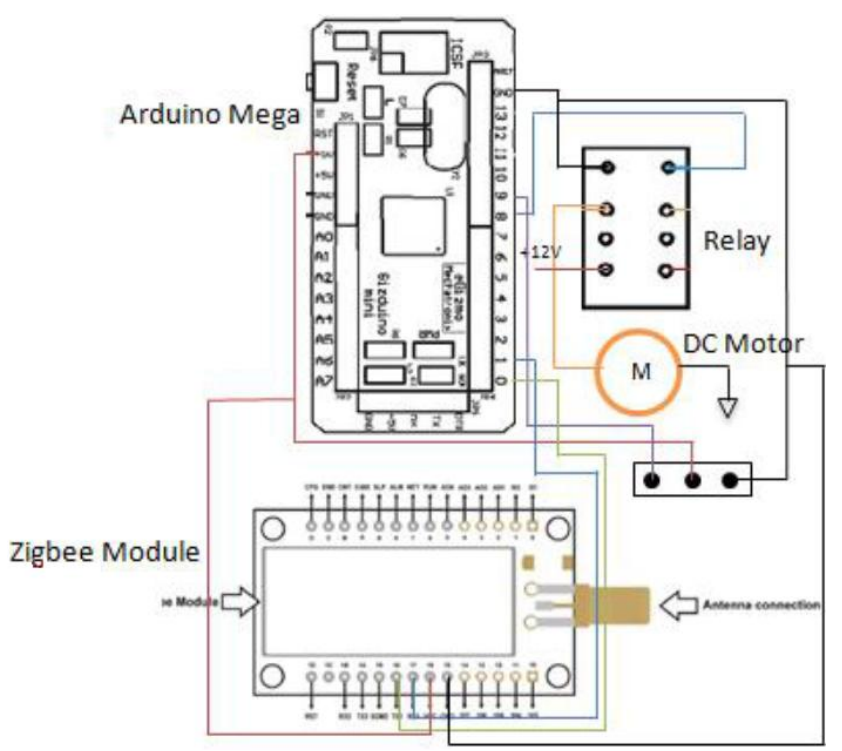

Figure 8. Circuit Diagram of the Luggage Cart 
Objective 2: To wirelessly control the luggage cart using two (2) Arduino Mega, two (2) Zigbee modules, and a direction/tilt sensor.

Programming the Arduino Mega of the user device: constint $\mathrm{A}=\mathrm{A} 6$; constint $\mathrm{B}=\mathrm{A} 7$;

int $\mathrm{Aval}=0$;

intBval $=0$;

void $\operatorname{setup}()$

\{

Serial.begin(9600);

Seriall.begin(9600);

pinMode(A, INPUT);

pinMode(B, INPUT);

\}

void $\operatorname{loop}()$

\{

Aval $=\operatorname{analog} \operatorname{Read}(\mathrm{A})$;

Bval = analogRead $(\mathrm{B})$;

// Serial.print(Aval);

// Serial.print(" ");

// Serial.println(Bval);

if (Aval < $500 \& \&$ Bval $<500)$

\{

Serial1.print('A');

\}

else if (Aval > $500 \& \&$ Bval $>500)$

\{

Serial1.print('B');

\}

else if(Aval > $500 \& \&$ Bval $<500)$

\{

Serial1.print('C');

\}

else if (Aval $<500 \& \&$ Bval $>500)$

\{

Serial1.print('D');

\}

Program the Arduino Mega of the luggage cart:

1. Do the wiring shown in Figure 7 and Figure 8 for the user device and the luggage cart.

2. Calibrate the tilt sensor, and the Zigbee module.

Objective 3: To navigate the wheels of the luggage cart with the use of a relay, dc motor, and servo motor.

1. Do the wiring for the relay and the DC motor shown in Figure 8.

2. Create a program for controlling the servo motor as stated below.

//Luggage Following Robot Receivera

\#include<Servo.h>

constintMotorPin = 7;

int state $=0$;

intpos = 90;

Servo dirServo;

charinData;

void $\operatorname{setup}()$

\{

pinMode(7, OUTPUT);

digitalWrite(7, LOW);

Serial.begin (9600);

dirServo.attach(10);

dirServo.write(90);
\}

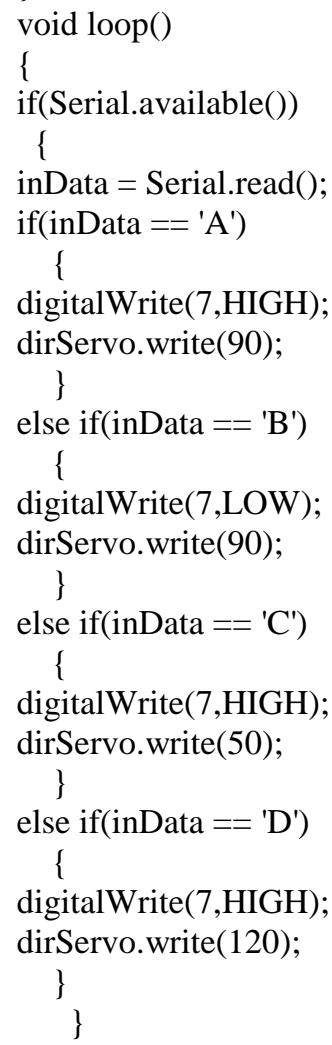

\{

if(Serial.available())

\{

inData $=$ Serial.read () ;

if $($ inData $==$ 'A')

\{

digitalWrite(7,HIGH);

dirServo.write(90);

\}

else if(inData $==$ 'B')

\{

digitalWrite(7,LOW);

dirServo.write(90);

\}

else if $\left(\right.$ inData $==$ 'C'$\left.^{\prime}\right)$

\{

digitalWrite(7,HIGH);

dirServo.write(50);

\}

else if $($ inData $==$ 'D')

\{

digitalWrite(7,HIGH);

dirServo.write(120);

\}

\subsection{Design Implementation}

The luggage cart is limited to a horizontal movement only. The wheels have only 90 degrees angle to turn left and right. There is at least 3 meters area wide enough to make the device turn. Neither it can climb stairs nor do any vertical movements. Its direction is limited only to forward, stop, left and right. It is neither waterproof nor can handle extreme shock. It can only carry a weight of less than or equal to 5.6 kilograms of luggage. The luggage cart has its own specified frequency of 2.454 $\mathrm{MHz}$ so that it will only follow its owner within a range of 50 meters. This is reflected as shown in Figure 9.

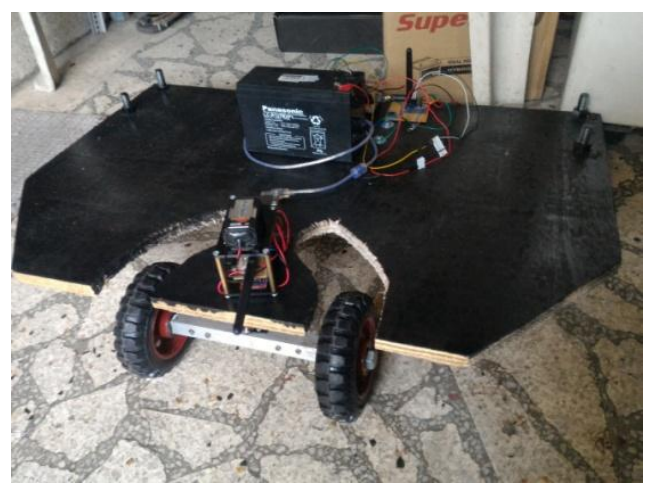

Figure 9. Actual Prototype of the System

\section{Conclusion and Recommendation}

\subsection{Conclusion}

The researchers conclude that the electronics design prototype is working. All of the objectives were met. They 
were able to create a luggage cart with two wheels at the back being controlled by the DC motor and two wheels in front being controlled by the servo motor. The two Zigbee modules and the two Arduino Mega microcontrollers were properly programmed and were able to give data.

\subsection{Recommendation}

If given the time and effort, the researchers will make a way to make the electronics design project portable and not bulky to the target client. A security system can also be added to secure and protect the luggage. Collision detection can also be installed. The design of the luggage can be improved to allow vertical movements as well.

\section{References}

[1] OSHA (2016). Baggage Tugs and Carts. Fundamentals Reference Guide. Retrieved from http://www.airlines.org/Documents/Baggage\%20Tugs\%20and\%2 0Carts.pdf. Accessed July 5, 2016.

[2] M. Bigos, J. D. LaRue, R. Mui and E. Wu (2011). "Final Project Report: Cart-A-Long".

[3] A. Savage and D. Ruko, "Kivi TV," Scripps TV Station Group, 7 March 2016. [Online]. Available:

http://www.kivitv.com/news/the-hit-list-3-smart-luggage-options. [Accessed 12 March 2016].
[4] D. Karastoyanov and M. Grouev (2011). "Wireless Controlled Luggage Carrier," Florida, USA

[5] M. DiGiacomcantonio and Y. Gebreyes. (2014). "Self-Propelled Luggage". United Sates of America Patent US 20140107868A1,

[6] Kannan, K. (2015). Arduino Based Voice Controlled Robot. International Research Journal of Engineering and Technology (IRJET), 2(1), 49-55.

[7] Aneiba, A., \&Hormos, K. (2014). A Model for Remote Controlled Mobile Robotic over Wi-Fi Network Using Arduino Technology. International Conference on Frontiers of Communications, Networks and Applications (ICFCNA 2014 - Malaysia), 1-4.

[8] Cuasito, R. J. (2013). Design, Development and Implementation of Educational and Entertainment Mobile Robots Utilizing Arduino Microcontroller, 11, 53-76.

[9] Balasubramanian, K., D, A. J. P., Chandrasekaran, A., V, N. P. M. \& Kiran, R. (2014). Efficient Mechanisms using ARDUINO to Control Robots, 2(1), 562-568.

[10] Arduino (2016).Arduino Mega 2560 Specification.Retrieved from https://www.arduino.cc/en/main/arduinoBoardMega2560. Accessed March 2016

[11] Arduino (2016). Zigbee Shield for Arduino. Retrieved from https://www.arduino.cc/en/Main/ArduinoXbeeShield Accessed March 2016.

[12] Tilt Sensor (2016). How do Tilts Sensor Works? Retrieved from http://www.azosensors.com/article.aspx?ArticleID=318.Accessed June 2016.

[13] Servo Motor (2016). Zigbee Shield for Arduino. Retrieved from https://www.allaboutcircuits.com/projects/servo-motor-controlwith-an-arduino/ Accessed July 2016.

[14] Relay (2016). HOW TO SET UP A 5V RELAY ON THE ARDUINO. http://www.circuitbasics.com/setting-up-a-5v-relayon-the-arduino/. Accessed July 2016. 